\title{
Theoretical Hypothesis of a Double Barrier Regarding the D-D Interaction in a Pd Lattice: A Possible Explanation of Cold Fusion Experiment Failures
}

\author{
Fulvio Frisone \\ Department of Physics, University of Catania, Catania I-95125, Italy
}

Received: June 06, 2013 / Accepted: August 12, 2013 / Published: January 31, 2014.

\begin{abstract}
During the past 15 years, disputable experimental evidence has built up for LERN (low energy nuclear reaction) phenomena in specialized heavy hydrogen systems. Actually, it can not be said that a new branch of science is beginning. In spite of experimental contributions, the real problem is that the theoretical statements of LERN are unknown. In this work, the authors analyze the deuteron-deuteron reactions within palladium lattice by means of the coherence theory of nuclear and condensed matter and, using this general theoretical framework accepted from "cold fusion scientists", it will be shown the low occurrence probability of fusion phenomena. In fact, in the coherence approach, the D-D potential exhibits double barrier features and, in this way, the D-D fusion is hampered.
\end{abstract}

Key words: Condensed matter, cold fusion, coherence theory.

\section{Introduction}

It has been studied that within the coherence theory of condensed matter, the cold fusion [1-4] phenomena was verified.

In Ref. [5], it is assumed that the e.m. (electromagnetic) field, due to elementary constituents of matter (i.e., ions and electrons) plays a very important role on dynamic system.

In fact, considering the coupling between e.m. equations, because of charged matter, and the Schrödinger equation of field matter operator, it is possible to demonstrate that in proximity of e.m. frequency $\omega_{0}$, the matter system shows a dynamic coherence. That is why it is possible to speak about the coherence domains whose length is about $\lambda_{C D}=2 \pi / \omega_{0}$.

Obviously, the simplest model of matter with coherence domain is the plasma system.

Corresponding author: Fulvio Frisone, Dr., research fields: low-energy fusion, dislocation of ions within a metal, many-body systems, the thermodynamic coherence of the material under stress. E-mail: fulvio.frisone@ct.infn.it.
In the usual plasma theory, it has to be considered the plasma frequency $\omega_{p}$ and the Debey length that measures the Coulomb force extension, i.e., the coherence domain length.

In order to address the crucial issue of the nuclear, fusion reaction involving the deuterons that pack the Pd-lattice, people must have a rather detailed understanding of the environment in which such nuclear process will eventually take place.

For a system with $N$, charge $Q$ and mass $m$ within a $V$ volume the plasma frequency can be written as:

$$
\omega_{\vec{k}}=\omega_{p}=\frac{Q e}{\sqrt{m}} \sqrt{\frac{N}{V}}
$$

Introducing the dimensional variable $\tau=\omega_{p} t$, the above equations can be rewritten:

$$
\begin{gathered}
\dot{\phi}_{\vec{n}}(\tau)=\frac{1}{2} \sum_{|\vec{k}|=\omega_{p}} \sum_{\vec{n}^{\prime} r}\left\langle\vec{n}\left|\alpha_{k r} \vec{\varepsilon}_{k r} \vec{a}^{+}-\alpha_{k r}^{*} \vec{\varepsilon}_{k r} \vec{a}\right| \vec{n}^{\prime}\right\rangle \phi_{\vec{n}^{\prime}}(\tau) \\
\frac{1}{2} \ddot{\alpha}_{k r}-i \dot{\alpha}_{k r}+m \lambda \alpha_{k r}=\frac{i}{2} \vec{\varepsilon}_{k r}^{*} \sum_{\vec{n} \vec{n}^{\prime}}\left\langle\vec{n}|\vec{a}| \vec{n}^{\prime}\right\rangle \phi_{\vec{n}}^{*}(t) \phi_{\vec{n}^{\prime}}(\tau)
\end{gathered}
$$

which, defining the state: 


$$
|\phi\rangle=\sum_{\vec{n}^{\prime}} \phi_{\bar{n}}(\tau)|\vec{n}\rangle
$$

and the e.m. field amplitude:

$$
\vec{A}=\sqrt{\frac{3}{8 \pi}} \sum_{r} \int d \Omega_{k} \alpha_{k r} \vec{\varepsilon}_{k r}
$$

re-writes:

$$
\begin{array}{r}
\left.\frac{\partial}{\partial t}|\phi\rangle=\sqrt{\frac{2 \pi}{3}}\left(\vec{A} \vec{a}^{+}-\vec{A}^{+} \vec{a}\right) \phi\right\rangle \\
\dot{\vec{A}}+\frac{i}{2} \ddot{\vec{A}}+i m \lambda \vec{A}=-\sqrt{\frac{2 \pi}{3}}\langle\phi|\vec{a}| \phi\rangle
\end{array}
$$

If only concentrate on one short time dynamics re-write:

$$
|\phi(0)\rangle=|0\rangle
$$

then, creating a difference in Eq. (6) and using Eq. (7) with $|\phi\rangle=|0\rangle$, can easily obtain:

$$
\begin{gathered}
\ddot{A}_{j}+\frac{i}{2} \dddot{A}_{j}=-\left(\frac{2 \pi}{3}\right)\left\langle 0 \mid\left[a_{j}, a_{l}^{+}\right] 0\right\rangle \\
A_{l}=-\left(\frac{2 \pi}{3}\right) A_{j}
\end{gathered}
$$

They have the same form of the equations of the coherence domains in the case in which:

$$
\begin{gathered}
\mu=0 \\
\lambda=0 \\
g^{2}=\left(\frac{2 \pi}{3}\right) \\
g_{c}^{2}=\frac{16}{27}<\frac{2 \pi}{3}
\end{gathered}
$$

Then, a solution for the ideal plasma exists. At any rate defining:

$$
\begin{aligned}
& \alpha_{k}=\left\langle\phi\left|a_{k}\right| \phi\right\rangle \\
& g_{0}=\left(\frac{2 \pi}{3}\right)^{1 / 2}
\end{aligned}
$$

In this case, the coupling critical constant is: the system is:

$$
\begin{gathered}
\dot{\alpha}_{k}=g_{0} A_{k} \\
\dot{A}_{k}+\frac{i}{2} \ddot{A}_{k}=-g_{0} \alpha_{k}
\end{gathered}
$$

so to admit the following holding quantity:

$$
Q=\sum\left\{A_{k}^{*} A_{k}+\frac{i}{2}\left(A_{k}^{*} \dot{A}_{k}-\dot{A}_{k}^{*} A_{k}\right)+\alpha_{k}^{*} \alpha_{k}\right\}
$$

while for the Hamiltonian's it is easy to compute:

$$
\frac{E}{N \omega_{p}}=H=Q+\sum\left[\frac{1}{2} \dot{A}_{k}^{*} \dot{A}_{k}-i g\left(A_{k}^{*} \alpha_{k}-A_{k} \alpha_{k}^{*}\right)\right]
$$

With the objective of seeing if there are any externals solutions, re-write:

$$
\begin{aligned}
& \alpha_{k}=\alpha u_{k} e^{i \psi} \\
& A_{k}=A u_{k} e^{i \phi}
\end{aligned}
$$

where, $\alpha$ and $A$ are positive constants and $u_{k}$ is a complex vector.

Changing these ones in Eqs. (12) and (13), then have:

$$
\begin{gathered}
\phi-\psi=\frac{\pi}{2} \\
\alpha=g_{0} \frac{A}{\dot{\phi}} \\
\frac{1}{2} \dot{\phi}^{3}-\dot{\phi}^{2}+g_{0}^{2}=0
\end{gathered}
$$

and by the condition $Q=0$ (can not have a net charge flow in a plasma), have:

$$
1-\dot{\phi}+\frac{g_{0}^{2}}{\dot{\phi}^{2}}=0
$$

In this case, it is easy to observe that for $g_{0}=\left(\frac{2 \pi}{3}\right)^{1 / 2}$ is unlikely to satisfy both the Eqs.

and (19).

This result means that the energy of an ideal quantum plasma does not have a minimum; in other words, the ideal quantum plasma does not exist.

That is not surprising because the Hamiltonian's describes, of this plasma, a system whose amplitude oscillations are arbitrary, while the limit over a certain amplitude does not exist in a real plasma.

But by:

$$
\vec{\xi}=\frac{1}{\sqrt{2 m \omega_{p}}}\left(\vec{a}+\vec{a}^{+}\right)
$$

it is easy to obtain:

$$
\left\langle\vec{\xi}^{2}\right\rangle=\frac{1}{m \omega_{p}} \alpha^{2}
$$

But in the plasma approximation as an homogeneous 
fluid, we suppose that our Hamiltonian's stops have to be valid for the oscillations bigger than the following:

$$
\left\langle\vec{\xi}^{2}\right\rangle_{\max }^{1 / 2} \approx a=\left(\frac{V}{N}\right)^{1 / 2}
$$

That is when the plasma's oscillations are of the same order of the inter-particle distance $a$.

In order to create some more realistic models of plasma, we want to compute the breaking amplitude $\alpha_{\max }$ obtained by the combination of the Eqs. (22) and (23) for a gas of electrons.

Using the definition of $\omega_{p}$, one have:

$$
\alpha_{\max }=\sqrt{m \omega_{p}} \cdot\left(\frac{1}{3}\right)^{1 / 3}=(m a)^{1 / 4} e^{1 / 2}
$$

Taking $\alpha \approx 2.5 \AA$, it is calculated: $\alpha_{\max } \cong 2.7 \AA$.

This simple calculation shows how it is possible to change the quantum ideal plasma in a real plasma.

For a plasma of electrons, the oscillations remain very little and so a two levels model can be a good approximation (the dynamics only includes the first excited state).

A consequence of this approximation, consisting in the reduction of the plasma in a homogeneous fluid, is the changing of the plasma frequency $\omega_{p}$ :

$$
\omega_{p}=\frac{Q}{\sqrt{m}} \sqrt{\frac{N}{V}}
$$

Moreover, one studies the "nuclear environment", that it is supposed existent within the palladium lattice $D_{2}$-loaded and at room temperature as predicted by coherence theory. In fact, when the palladium lattice is loaded with deuterium gas, some physicists declared that it is possible to observe traces of nuclear reactions [1-3]. For this reason, many of these physicists speak about LERN (low energy nuclear reaction).

One of the biggest experiments tells people that in the $D_{2}$-loaded palladium case, the most frequent nuclear reactions are $[3,4]$ :

(1) $\mathrm{D}+\mathrm{D} \rightarrow{ }^{3} \mathrm{H}+\mathrm{p}+4.03 \mathrm{MeV}$

(2) $\mathrm{D}+\mathrm{D} \rightarrow{ }^{4} \mathrm{He}+$ gamma $(23.85 \mathrm{MeV})$

In this work, aim to propose a "coherence" model by means of which can explain the occurrence of reactions (1) and (2) and their probability according to the most reliable experiments [6]. First of all, the author will start by analysing the environment, i.e., of plasmas present within palladium (d-electron, s-electron, Pd-ions and D-ions) using the coherence theory of matter; lastly the effective potential reported in Refs. [7, 8] will be used, adding the role of lattice perturbations by means of which the $D-D$ tunneling probability is computed.

\section{The Plasmas Present within no Loaded Palladium}

According to the coherence theory of condensed matter, in a Pd crystal at room temperature the electron shells are in a coherent regime within coherent domain. In fact, they oscillate in tune with a coherent e.m. field trapped in the coherent domains.

So the authors must take the plasma of s-electron and d-electron into account, in order to describe the lattice environment.

\subsection{The Plasma of the d-electrons}

Similar argumentations were proposed by Preparata's but started from a new formulation of condense matter theory known as coherence theory.

In this theory, it can be visualized the plasma formed by d-shell electrons as consisting of charged shells of charge $n_{d} e$ (for palladium $n_{d}=10$ ) radius $r_{d}=1 \AA$ and thickness a fraction of one Angstrom. The classical plasma:

$$
\omega_{d}=\frac{e}{\sqrt{m}} \sqrt{\frac{n_{d} N}{V}}
$$

as d-electrons plasma frequency. But according to the coherence theory of matter, we must adjust this plasma frequency of a factor 1.38.

It can be understood this correction by observing that the formula (26) is obtained assuming a uniform d-electron charge distribution. But of course the d-electron plasma is localized in a shell of radius $R$ (that is about $1 \AA$ ), so the geometrical contribution is:

$$
\sqrt{\frac{6}{\pi}}=1.38
$$

If re-write it with $\omega_{d}$, the renormalized d-electron plasma frequency, one have: 


$$
\omega_{d}=41.5 \mathrm{eV} / \hbar
$$

and the maximum oscillation amplitude $\xi_{d}$ is about $0.5 \AA$.

\subsection{The Plasma of Delocalized s-electrons}

The s-electrons are those which in the lattice neutralize the adsorbed deuterons ions. They are delocalised and their plasma frequency depends on loading ratio ( $D / P d$ percentage). Eq. (28) can also be written as:

$$
\omega_{s e}=\frac{e}{\sqrt{m}} \sqrt{\frac{N}{V}} \cdot \sqrt{\frac{x}{\lambda_{a}}}
$$

where:

$$
\lambda_{a}=\left[1-\frac{N}{V} V_{p d}\right]
$$

and $V_{p d}$ is the volume effectively occupied by the Pd-atom. As reported in Ref. [5], one obtains:

$$
\omega_{s e} \approx x^{1 / 2} 15.2 \mathrm{eV} / \hbar
$$

For example, for $x=0.5$, one has $\omega_{s e} \sim 10.7 \mathrm{eV} / \hbar$.

\subsection{The Plasma of Pd-ions}

Further, it can be considered the plasma due to the Palladium ions forming the lattice structure; in this case, it is possible to demonstrate that the frequency is Eq. (28):

$$
\omega_{p d}=0.1 \mathrm{eV}
$$

\section{The Plasmas Present within $D_{2}$-Loaded Palladium}

In this section, the author seek to show what happens when the absorbed deuterium is placed near to the palladium surface. This loading can be enhanced using electrolytic cells or vacuum chambers working at opportune pressure [9-11]. By means of Preparata's theory of condensed matter, it is assumed that according to the ratio $x=D / P d$, there are three phases concerning the $\mathrm{D}_{2}-\mathrm{Pd}$ system:

- phase $\alpha$ for $x<0.1$;

- phase $\beta$ for $0.1<x<0.7$;

- phase $\gamma$ for $x>0.7$.
In the $\alpha$-phase, the $D_{2}$ is in a disordered and not coherent state ( $D_{2}$ is not charged).

According to the other phases, people start remembering that on the surface, because of the lattice e.m., takes place the following reaction:

$$
D_{\text {attice }} \rightarrow D^{+}+e^{-}
$$

Then, according to the loading quantity $x=D / P d$, the ions of deuterium can occur on the octahedral sites (Fig. 1) or on the tetrahedral (Fig. 2) in the (1, 0, 0 )-plane. In the coherent theory of the so-called $\beta$-plasma of Preparata's the deuterons plasma are in the octahedral site and the $\gamma$-plasma are in the tetrahedral.

Regarding to the $\beta$-plasma, it is possible to affirm that the plasma frequency is given by Eq. (28):

$$
\omega_{\beta}=\omega_{\beta 0}(x+0.05)^{1 / 2}
$$

where:

$$
\omega_{\beta 0}=\frac{e}{\sqrt{m_{D}}}\left(\frac{N}{V}\right)^{1 / 2} \frac{1}{\lambda_{a}^{1 / 2}}=\frac{0.15}{\lambda_{a}^{1 / 2}} \mathrm{eV} / \hbar
$$

For example, if use $\lambda_{a}=0.4$ and $x=0.5$, one obtains $\omega_{\beta}=0.168 \mathrm{eV} / \hbar$.

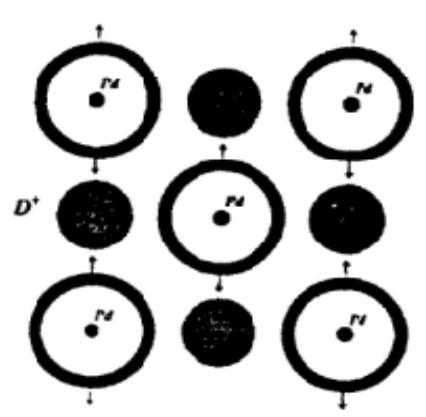

Fig. 1 The octahedral sites of the Pd lattice where the deuterons take place.

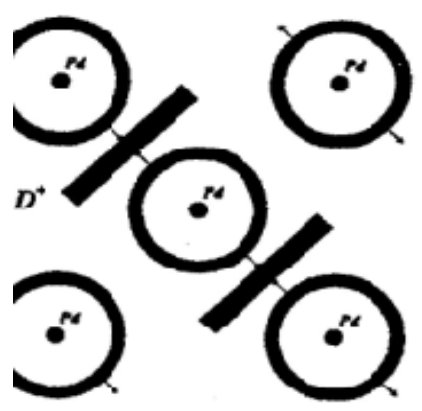

Fig. 2 The thin disks of the tetrahedral site of the Pd lattice where the deuterons take place. 
In the tetrahedral sites, the $D^{+}$can occupy the thin disk that encompass two sites (Fig. 3), representing a barrier to the $D^{+}$ions. The author must underline that the electrons of the $d$-shell start oscillating near to the equilibrium distance $y_{0}$ (about $1.4 \AA$ ) so that the static ions have a cloud of negative charge [5]. Then follows:

$$
\omega_{\gamma}=\sqrt{\frac{4 Z_{e f f} \alpha}{m_{D} y_{0}^{2}}} \approx 0.65 \mathrm{eV} / \hbar
$$

Of course, this frequency also depends by the chemical condition of the palladium (impurities, temperature etc...).

Due to a large plasma oscillation of d-electrons, in the disk-like tetrahedral region (where the $\gamma$-phase $D^{+}$, s are located) condenses a high density negative charge giving rise to a screening potential $W(t)$ whose profile is reported in Fig. 4.

Having mapped that the $\gamma$-phase depends on $x$ value other can experimentally observe the new phase in Refs. $[11,12]$ very important in the (LERN) investigation. This is demonstrated by the fact that many of the "cold fusion physicist" declare that the main point of cold fusion protocol is the loading $D / P d$ ratio higher than 0.7 , i.e., the deuterium takes place in the tetrahedral sites.

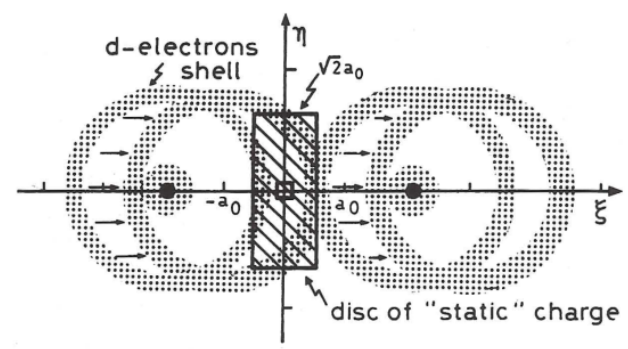

Fig. 3 Possible d-electron plasma oscillation in a Pd lattice.

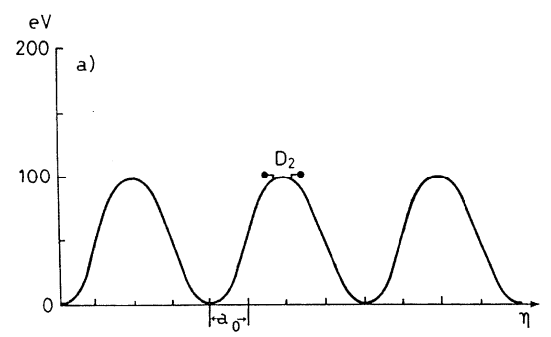

Fig. 4 The profile of the electrostatic potential in a arbitrarily direction $\eta$.

\section{The D-D Potential}

In Ref. [7], it was shown that the phenomenon of fusion between nuclei of deuterium in the lattice of a metal is conditioned by the structural characteristics, by the dynamic conditions of the system and also by the concentration of impurities present in the metal under examination.

In fact, studying the curves of the potential of interaction between deuterons (including the deuteron-plasmon contribution) in the case of three typical metals ( $P d, P t$ and $T i)$, as shown in a three-dimensional model, the height of the Coulomb barrier decreases on the varying of the total energy and of the concentration of impurities present in the metal itself.

The initial potential that connects the like-Morse attraction and the like-Coulomb repulsion can be written as seen in Refs. [7, 8]:

$$
V(r)=k_{0} \frac{q^{2}}{r}\left(V_{M}(r)-\frac{\Sigma}{r}\right)
$$

where, $V(r)_{M}$ is the Morse potential, $k_{0}=\left(1 / 4 \pi \varepsilon_{0}\right)$, $q$ is the charge of the deuteron, $M_{d}$ is the reduced mass of the deuterium nuclei, $T$ is the absolute temperature at which the metal is experimentally placed, $J$ is the concentration of impurities in the crystalline lattice and $R$ is the nuclear radius.

In Eq. (37), $V(r)_{M}$ is a like-Morse potential, and is given by:

$$
V(r)_{M}=B\left\{\exp \left(-2 \varphi\left(r-r_{0}\right)\right)-2 \exp \left(-\varphi\left(r-r_{0}\right)\right)\right\}
$$

Here the parameters $B, \varphi$ ed $r_{0}$ depend by the lattice.

In fact, the potential (37) is an effective one whose reliability is demonstrated by its ability to fit the Coulomb potential for $r \rightarrow 0$ and the Morse potential in the attractive zone. So, Siclen and Jones [12] define $\rho$ the point where the Coulomb potential is associated to the Morse trend, $r^{\prime}{ }_{0}$ the equilibrium distance and $D$ ' the well.

Of course, in the free space for a $D_{2}$ molecule, $\rho$ is about $0.3 \AA, r^{\prime}{ }^{\prime}$ is about $0.7 \AA$ and $D^{\prime}$ is $-4.6 \mathrm{eV}$. 
But within the lattice there are the screening effect and the deuteron-deuteron interaction, by means of phonon exchange, modify these parameter values.

Since the screening effect can be modulated by the giver atoms, considering Refs. [7, 8], the role of impurities and it has been shown that can be written:

$$
J=J_{0} \exp \left[\frac{\beta}{b k T}\right] .
$$

Further, some particular reactions could occur, incorporating the impurities in the nucleus of the dislocations, as a result of the different arrangement of the atoms with respect to that of the unperturbed lattice.

This type of process has been extensively studied in the literature for metals and for the case of crystalline semiconductors at high temperature.

In the latter, for example, it is found that the concentration of interstitial impurities around a linear dislocation, with a point component, depends on the temperature according to the law written above where $J_{0}$ is the concentration of impurities in the zone with zero internal pressure, $\left(b^{3} \simeq v_{i}\right)$ the volume of the ions constituting the lattice, while $\beta$ is proportional to the difference $\left(v_{d}-v_{i}\right)$ between the volume of the impurity atoms and that of the lattice ions.

The author's conjecture is that in a metal, such as Pd, a similar phenomena could occur between the atoms of deuterium penetrating the lattice as a result of deuterium loading and the micro-cracks produced by variations in temperature. In this case, the parameter $\beta$ of the previous expression would be negative, determining an increase in the concentration of deuterons in the vicinity of the micro-crack, which would then catalyse the phenomenon of fusion as:

$$
\Sigma=J K T R
$$

and:

$$
B=J / \zeta
$$

So the effective $d-d$ potential can be wrote:

$$
V(r)=k_{0} \frac{q^{2}}{r} \cdot\left(V(r)_{M}-\frac{J K T R}{r}\right)
$$

where, $V(r)_{M}$ is the Morse potential, $k_{0}=\left(1 / 4 \pi \varepsilon_{0}\right)$, $q$ is the charge of the deuteron, $M_{d}$ is the reduced mass of the deuterium nuclei, $T$ is the absolute temperature at which the metal is experimentally placed, $J$ is the concentration of impurities in the crystalline lattice and $R$ is the nuclear radius.

Considering the attractive force, due to the exchange of plasmons, the main contributions are those:

Taking into account the role of coupling between deuteron and plasmons, in Ref. [13], it was numerically evaluated a $D-D$ potential having the features of the potential (14) with $D^{\prime}=-50 \mathrm{eV}$ and $r^{\prime}{ }_{0}=0.5 \AA$ and $\rho=$ $0.2 \AA$ (remember that in Ref. [13] are considered only two plasmon excitations at $7.5 \mathrm{eV}$ and $26.5 \mathrm{eV}$ ).

The feature of the potential (37) is shown as below:

According to the coherence theory of condensed matter, I study the role of potential (41) in the three different phases : $\alpha, \beta$ and $\gamma$.

This section, however, needs of some important explanation, such as:

- What $K T$ is;

- What is the role of electrons, ions plasma?

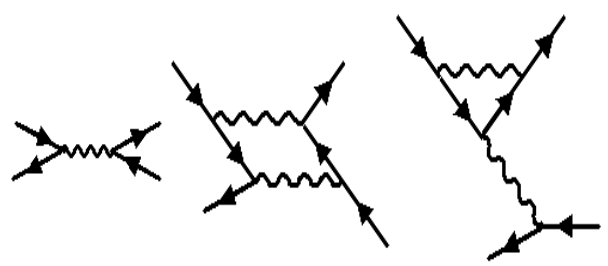

Fig. 5 Plasmon exchanges. Solid lines indicate deuterons and wiggly lines plasmons.

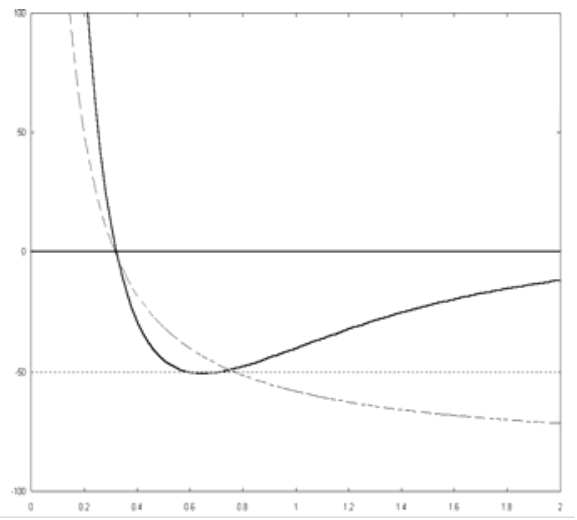

Fig. 6 The solid line shows the features of potential (37) computed in order to obtain $D^{\prime}=-50 \mathrm{eV}$ and $\rho=0.165 \AA$. The coulomb potential in dashed line is computed using a screening constant of $85 \mathrm{eV}$. In the $\mathrm{x}$-axes, it is reported the distance in Bohr radius unit and on the $y$-axes the energy in eV. 
Concerning with the first point, according to the different deuteron-lattice configurations, $K T$ can be:

- the loaded lattice temperature if we consider;

- the deuterons in the $\alpha$-phase;

- $\omega_{\beta}$ if the deuterons in the $\beta$-phase is considered;

- $\omega_{\gamma}$ if the deuterons in the $\gamma$-phase is considered.

The question is much more complicate for the second point. In fact, the lattice environment is a combination of coherent plasmas (ion Pd, electron and deuterons plasma) at different temperature, due to different masses, that let be very difficult the description of the emerging potential.

The method that in this work we propose is the following: to consider the total screening contribution of lattice environment at D-D interaction (i.e., $V_{t o t}$ ) as random potential $Q(t)$. So in this model one has:

$$
V_{t o t}(t)=V(r)+Q(t)
$$

Of course, assume that:

$$
\langle Q(t)\rangle_{t} \neq 0
$$

That is to say that $Q(t)$ (a second order potential contribution) is a periodic potential (the frequency will be called by $\omega_{Q}$ ) that oscillates between the maximum value $Q_{\max }$ and 0 .

More exactly the oscillation charges of $d$-shell produce a screening potential having a harmonic feature:

$$
e V(r)=-Z_{d} \frac{k e^{2}}{2 a_{0}} r^{2}
$$

In Ref. [5], putting $Z_{d}=10 / 3$ and $a_{0}=0.7 \AA$, it is evaluated a screening potential $V_{0}$ of about $85 \mathrm{eV}$. In this way, we can compute $\rho=V_{0} / 26.9$ and at last $\rho=$ $0.165 \AA$. To summarize, I can have the following cases in a palladium lattice according to the loading ratio:

(1) $\alpha$-phase

In the phase $\alpha$, the deuterons are in a molecular state and the thermal motion is about:

$0.02 \mathrm{eV}<\hbar \omega_{\alpha}<0.1 \mathrm{eV}$

This phase takes places when $x$ is fewer than 0.1 , and since $W(t)$ is zero, the $\mathrm{D}$ - $\mathrm{D}$ potential is:

$$
V(r)=k \frac{q^{2}}{r} \cdot\left(V_{M}(r)-\frac{J \hbar \omega_{\alpha} R}{r}\right)
$$

Eq. (45) was partially evaluated in Ref. [7]; in fact, people were only interested in the dependence of the tunneling probability on impurities present within lattice. Through this work, the author seek to examine the correlation between potential features and loading ratio and in the Paragraph 5, the authors showed some numerical results.

(2) $\beta$-phase

When $x$ is bigger than 0.1 but smaller than 0.7 , the phase $\beta$ happens. The interaction takes place among deuteron ions that oscillate between the following energy values:

\section{$0.1 \mathrm{eV}<\hbar \omega_{\beta}<0.2 \mathrm{eV}$}

In this case, $W(t)$ is zero, give this expression to the potential:

$$
V(r)=k \frac{q^{2}}{r} \cdot\left(V_{M}(r)-\frac{J \hbar \omega_{\beta} R}{r}\right)
$$

Comparing with Eqs. (45) and (46), it seems clear that the weight of impurities is more important in the $\beta$-phase. Of course, this conclusion deals with the previous papers $[7,8]$ in which the authors studied the role of temperature on tunneling effect.

(3) $\gamma$-phase

Lastly, when the loading ratio is higher than 0.7 , the deuteron-palladium system is in the phase $\gamma$.

This is the most interesting case. The deuterons cross the screening through the d-electrons shell, in this sense, the authors did a numeric simulation where I suppose that the D-D potential must be computed assuming that the well present in potential (37), because of the Morse contribution, disappears. In fact if a classical plasma model is used where the $D^{+}$ions are the positive charge and the d-electrons the negative one, it is very "realistic" to use the following potential:

$$
V(r, t)=k \frac{q^{2}}{r} \cdot\left(V_{M}(r)-\frac{J \hbar \omega_{\gamma} R}{r}\right)+Q(t)
$$

where, $Q(t)$ is a not known perturbative potential. About this it can be also added that:

$$
\langle Q(t)\rangle_{t} \approx \frac{W_{\max }}{\sqrt{2}}
$$


As said previously, the authors suppose that it is the screening potential, because of the d-electrons, has the role to reduce the repulsive barrier, i.e, $\rho$ and $r_{0}{ }_{0}$. In the next evaluation, it is given:

$$
\langle Q(t)\rangle_{t} \approx 85 \mathrm{eV}
$$

\section{Conclusions}

The aim of this section is to present the D-D fusion probability normalized to number of events per second regarding the D-D interaction in all different phases. More exactly, authors seek to compare, at the varying of energy between $-50 \mathrm{eV}$ to $50 \mathrm{eV}$, the fusion probability in the phase $\alpha, \beta$ and $\gamma$. The role of d-electrons screening as perturbative lattice potential is also considered.

This treatment, which only interests the case where $Q(t)$ is different from zero, involves the change of the value of the point on the $x$-axes where the Coulomb barrier takes place; in this case, the final result is that the screening enhances the fusion probability.

In order to evaluate the fusion rate $(\Lambda)$, this formula is applied:

$$
\Lambda=\mathrm{A} \Gamma
$$

where, $\Gamma$ is the Gamow factor and $A$ is the nuclear reaction constant obtained from measured cross sections (value used was $10^{22} \mathrm{~s}^{-1}$ ).

From an experimental point of view, in the cold fusion phenomenology, it is possible to affirm that there are three typologies of experiments [14]:

- those giving negative results;

- those giving some results (little detection signs with respect to background, fusion probability about $10^{-23}$ using a very high loading ratio);

- those giving clear positive results such as the Fleischmann and Pons experiments.

Nevertheless, the authors think that from the experimental point of view, the experiments of the third point are few accurate. In this sense, we believe that a theoretical model of controversial phenomenon of cold fusion, is better to explain only those experiments of the points 1 and 2. In this case, it is important to consider the role of loading ratio on the experimental results. Table 1 is shown the results about the $\alpha$-phase. Here, it can be observed that the theoretical fusion probability is very low, smaller than $10^{-74}$. Consequently, it is allowed to affirm that through the loading, in the deuterium, of a percentage of $x<0.2$ it is not possible for any fusion. The same absence of nuclear phenomenon is compatible for a loading ratio of about 0.7 (Table 2) since in this case the predicted fusion probability is smaller than $10^{-42}$. These predictions, of course, agree with the experimental results (for $x>0.7$ look at Ref. [6]). The result of the model is that in the $\gamma$-phase, where some background fluctuations can be observed, because of a high loading ratio, the authors predict a fusion probability at about $10^{-22}$. This is a new result, very important for Refs. [7, 8] since, in those cases, the fusion probability was independent from the loading ratio. To conclude, the aim is also to show that the model proposed in this paper (which unify nuclear physics with condensed matter) can explain some irregular nuclear traces in the solids. Regarding the experiments at point 3, on the other hand, supposing that those could be real, we want to consider other contributions as micro-deformation occurrence in order to explain the very high fusion rate. The role of micro-crack and impurities linked by loading ratio will be explored in other speculative works. "The nuclear physics within condensed matter" may be a new device through which it is possible to understand other new productive scientific topic.

The studied problem is the objective of proposing a theoretical model considering the random city of the phenomenon and that permits to those experiments (in which there is a high production of energy) to be repetitive and reproductive.

The starting point is the following: the phenomenon of the low-energy fusion critically, depends on the interaction between the $\mathrm{d}-\mathrm{d}$ nuclear system and the $\mathrm{D}_{2}$ gas system, and the lattice of the Palladium.

In fact, at the variation of the loading percentage, the typical deuterium-plasma interaction of the $\gamma$ phase grows up and so the electrostatic repulsion is weakened. 
Table 1 Using the $\alpha$-phase potential (potential 45 ), the fusion probability has been computed for Pd "Impure" $(J \approx 0.75 \%)$, normalized to the number of event/sec for different values of energy $(-50 \mathrm{eV}<E<50 \mathrm{eV})$.

Palladium $J \approx 0.75 \% ; \rho \approx 0.34 ; \AA r^{\prime}{ }_{0}=0.7 ; \AA \mathrm{D}^{\prime}=-50 \mathrm{eV}$.

\begin{tabular}{llll}
\hline$\omega_{\alpha} \approx 0.0025 \mathrm{eV}$ & $\omega_{\alpha} \approx 0.05 \mathrm{eV}$ & $\omega_{\alpha} \approx 0.075 \mathrm{eV}$ & $\omega_{\alpha} \approx 0.1 \mathrm{eV}$ \\
\hline$E \approx-50 P \approx 10^{-100}$ & $E \approx-50 P \approx 10^{-103}$ & $E \approx-50 P \approx 10^{-100}$ & $E \approx-50 P \approx 10^{-99}$ \\
$E \approx-40 P \approx 10^{-99}$ & $E \approx-40 P \approx 10^{-101}$ & $E \approx-40 \mathrm{P} \approx 10^{-98}$ & $E \approx-40 P \approx 10^{-97}$ \\
$E \approx-30 P \approx 10^{-97}$ & $E \approx-30 P \approx 10^{-100}$ & $E \approx-30 P \approx 10^{-96}$ & $E \approx-30 P \approx 10^{-96}$ \\
$E \approx-20 P \approx 10^{-95}$ & $E \approx-20 P \approx 10^{-99}$ & $E \approx-20 P \approx 10^{-94}$ & $E \approx-20 P \approx 10^{-93}$ \\
$E \approx-10 P \approx 10^{-94}$ & $E \approx-10 P \approx 10^{-97}$ & $E \approx-10 P \approx 10^{-91}$ & $E \approx-10 P \approx 10^{-90}$ \\
$E \approx 0 P \approx 10^{-92}$ & $E \approx 0 P \approx 10^{-96}$ & $E \approx 0 P \approx 10^{-90}$ & $E \approx 0 P \approx 10^{-86}$ \\
$E \approx 10 P \approx 10^{-91}$ & $E \approx 10 P \approx 10^{-94}$ & $E \approx 10 P \approx 10^{-87}$ & $E \approx 10 P \approx 10^{-83}$ \\
$E \approx 20 P \approx 10^{-90}$ & $E \approx 20 \mathrm{P} \approx 10^{-92}$ & $E \approx 20 \mathrm{P} \approx 10^{-85}$ & $E \approx 20 P \approx 10^{-80}$ \\
$E \approx 30 P \approx 10^{-89}$ & $E \approx 30 P \approx 10^{-90}$ & $E \approx 30 P \approx 10^{-82}$ & $E \approx 30 P \approx 10^{-78}$ \\
$E \approx 40 P \approx 10^{-86}$ & $E \approx 40 P \approx 10^{-89}$ & $E \approx 40 P \approx 10^{-80}$ & $E \approx 40 P \approx 10^{-74}$ \\
$E \approx 50 P \approx 10^{-84}$ & $E \approx 50 P \approx 10^{-87}$ & $E \approx 50 P \approx 10^{-79}$ & $E \approx 50 P \approx 10^{-71}$
\end{tabular}

Table 2 Using the $\beta$-potential (potential 46), the fusion probability $P$ has been computed for Pd "Impure" $(J \approx 0.75 \%)$, normalized to the number of event/sec for different values energy $(-50 \mathrm{eV}<E<50 \mathrm{eV})$.

Palladium $J \approx 0.75 \% ; \alpha \approx 0.34 ; \AA \mathrm{r}^{\prime}{ }_{0}=0.7 ; \AA \mathrm{D}^{\prime}=-50 \mathrm{eV}$.

\begin{tabular}{llll}
\hline$\omega_{\beta} \approx 0.125 \mathrm{eV}$ & $\omega_{\beta} \approx 0.150 \mathrm{eV}$ & $\omega_{\beta} \approx 0.175 \mathrm{eV}$ & $\omega_{\beta} \approx 0.2 \mathrm{eV}$ \\
\hline$E \approx-50 P \approx 10^{-83}$ & $E \approx-50 P \approx 10^{-88}$ & $E \approx-50 P \approx 10^{-86}$ & $E \approx-50 P \approx 10^{-81}$ \\
$E \approx-40 P \approx 10^{-81}$ & $E \approx-40 P \approx 10^{-87}$ & $E \approx-40 P \approx 10^{-85}$ & $E \approx-40 P \approx 10^{-75}$ \\
$E \approx-30 P \approx 10^{-80}$ & $E \approx-30 P \approx 10^{-86}$ & $E \approx-30 P \approx 10^{-83}$ & $E \approx-30 P \approx 10^{-73}$ \\
$E \approx-20 P \approx 10^{-79}$ & $E \approx-20 P \approx 10^{-85}$ & $E \approx-20 P \approx 10^{-80}$ & $E \approx-20 P \approx 10^{-70}$ \\
$E \approx-10 P \approx 10^{-78}$ & $E \approx-10 P \approx 10^{-84}$ & $E \approx-10 P \approx 10^{-74}$ & $E \approx-10 P \approx 10^{-68}$ \\
$E \approx 0 P \approx 10^{-76}$ & $E \approx 0 P \approx 10^{-82}$ & $E \approx 0 P \approx 10^{-73}$ & $E \approx 0 P \approx 10^{-62}$ \\
$E \approx 10 P \approx 10^{-75}$ & $E \approx 10 P \approx 10^{-81}$ & $E \approx 10 P \approx 10^{-72}$ & $E \approx 10 P \approx 10^{-60}$ \\
$E \approx 20 P \approx 10^{-74}$ & $E \approx 20 P \approx 10^{-79}$ & $E \approx 20 P \approx 10^{-71}$ & $E \approx 20 P \approx 10^{-54}$ \\
$E \approx 30 P \approx 10^{-73}$ & $E \approx 30 P \approx 10^{-76}$ & $E \approx 30 P \approx 10^{-70}$ & $E \approx 30 P \approx 10^{-50}$ \\
$E \approx 40 P \approx 10^{-72}$ & $E \approx 40 P \approx 10^{-75}$ & $E \approx 40 P \approx 10^{-69}$ & $E \approx 40 P \approx 10^{-45}$ \\
$E \approx 50 P \approx 10^{-71}$ & $E \approx 50 P \approx 10^{-70}$ & $E \approx 50 P \approx 10^{-65}$ & $E \approx 50 P \approx 10^{-42}$
\end{tabular}

Table 3 Using the $\gamma$-potential (potential 47), the fusion probability has been computed for Pd "Impure" $(J \approx 0.75 \%)$, normalized to the number of event/sec for different values of energy $(-50 \mathrm{eV}<E<50 \mathrm{eV})$.

Palladium $J \approx 0.75 \% ; \rho \approx 0.165 ; \AA r^{\prime}{ }_{0}=0.35 ; \AA D^{\prime}=-50 \mathrm{eV}$.

\begin{tabular}{llll}
\hline$\omega_{\gamma} \approx 0.6 \mathrm{eV}$ & $\omega_{\gamma} \approx 0.65 \mathrm{eV}$ & $\omega_{\gamma} \approx 0.7 \mathrm{eV}$ & $\omega_{\gamma} \approx 0.75 \mathrm{eV}$ \\
\hline$E \approx-50 P \approx 10^{-71}$ & $E \approx-50 P \approx 10^{-51}$ & $E \approx-50 P \approx 10^{-57}$ & $E \approx-50 P \approx 10^{-50}$ \\
$E \approx-40 P \approx 10^{-68}$ & $E \approx-40 P \approx 10^{-49}$ & $E \approx-40 P \approx 10^{-54}$ & $E \approx-40 P \approx 10^{-47}$ \\
$E \approx-30 P \approx 10^{-66}$ & $E \approx-30 P \approx 10^{-47}$ & $E \approx-30 P \approx 10^{-51}$ & $E \approx-30 P \approx 10^{-44}$ \\
$E \approx-20 P \approx 10^{-62}$ & $E \approx-20 P \approx 10^{-44}$ & $E \approx-20 P \approx 10^{-49}$ & $E \approx-20 P \approx 10^{-41}$ \\
$E \approx-10 P \approx 10^{-60}$ & $E \approx-10 P \approx 10^{-41}$ & $E \approx-10 P \approx 10^{-46}$ & $E \approx-10 P \approx 10^{-39}$ \\
$E \approx 0 P \approx 10^{-57}$ & $E \approx 0 P \approx 10^{-39}$ & $E \approx 0 P \approx 10^{-43}$ & $E \approx 0 P \approx 10^{-36}$ \\
$E \approx 10 P \approx 10^{-54}$ & $E \approx 10 P \approx 10^{-38}$ & $E \approx 10 P \approx 10^{-42}$ & $E \approx 10 P \approx 10^{-33}$ \\
$E \approx 20 P \approx 10^{-51}$ & $E \approx 20 P \approx 10^{-35}$ & $E \approx 20 P \approx 10^{-40}$ & $E \approx 20 P \approx 10^{-32}$ \\
$E \approx 30 P \approx 10^{-49}$ & $E \approx 30 P \approx 10^{-32}$ & $E \approx 30 P \approx 10^{-36}$ & $E \approx 30 P \approx 10^{-29}$ \\
$E \approx 40 P \approx 10^{-47}$ & $E \approx 40 P \approx 10^{-30}$ & $E \approx 40 P \approx 10^{-33}$ & $E \approx 40 P \approx 10^{-25}$ \\
$E \approx 50 P \approx 0^{-45}$ & $E \approx 50 P \approx 10^{-27}$ & $E \approx 50 P \approx 10^{-30}$ & $E \approx 50 P \approx 10^{-21}$ \\
\hline
\end{tabular}

But while the deuterium loading in the gas phase grows up, the palladium lattice is deformed.

Other theoretical and experimental studies about the electric field are in progress. In order to be clearer, the main passages of the lattice reaction is explained: Firstly, we have the deformation; Secondly, the 
dislocation; and lastly the micro-crack.

If the lattice is deformed, within the micro-crack one has the creation of a microscopic electric field, in which the $D^{+}$and the plasmons come to collision, and in which the deuterium nuclei are accelerated, as it happens in a classic accelerator of nuclear physic. This acceleration can also generate the phenomenon of the low-energy fusion.

The reason why the author is taking the palladium is very simple first is very stable, another reason is that it has 10 electrons $\mathrm{d}$, for which makes it very conductive, so during the loading phase of deuterium, which has an absorption coefficient and very high compared to the diffusion of titanium and nickel.

An important aspect is that the structure of the titanium is hexagonal, also has only two electrons then de is not very stable. As to the nickel has a FCC structure such as palladium and is composed of $9 \mathrm{~d}$ electrons, so little "stable". While palladium has 10 electrons $\mathrm{d}$, I together with my collaborators we are thinking of making a new palladium which has a greater number of d electrons therefore very stable with a very high absorption coefficient. For reasons I can not reveal copyright methods with the traditional palladium because I am trying new techniques forced loading.

\section{References}

[1] T. Hayashi, H. Iwamura, M. Naito, Y. Matsumoto, Y. Uozumi, M. Miki, et al., Catalytic asymmetric reduction of allylic esters with formic acid catalyzed by palladium-MOP complexes, Japanese Journal of Applied
Physics 116 (1994) 775-776.

[2] Reifenschweiler, Reduced radioactivity of tritium in small titanium particles, Physics Letters 184 (1994) 149-153.

[3] Reifenschweiler, Cold fusion and decrease of tritium radioactivity, Fusion Technology 30 (1996) 261-263.

[4] H. Melvin, Anomalous effects involving excess power, radiation, and helium production during D2O electrolysis using palladium cathodes, Fusion Technology 25 (1994) 478-486.

[5] G. Preparata, QED Coherence in Matter, World Scientific Publishing, Singapore, 1995.

[6] S. Aiello, Nuclear fusion experiment in Pd charged by deuterium gas, Fusion Technology 18 (1990) 125-132.

[7] F. Frisone, Theoretical model of the probability of fusion between deuterons within deformed crystalline lattices with microcracks at room temperature, Fusion Technology 40 (2001) 139-146.

[8] F. Frisone, Deuteron interaction within a microcrack in a lattice at room temperature, Fusion Technology 39 (2001) 260-265.

[9] M. Fleishmann, S. Pons, Electrochemically induced nuclear fusion of deuterium, J. Electroanal. Chem. 261 (1989) 301-308.

a) de Ninno, Evidence of emission of neutrons from a titanium-deuterium system, Europhysics Letters 9 (1989) 221-224.

[10] G. Mengoli, The coulomb barrier not static in QED a correction to the theory by preparata on the phenomenon of cold fusion and theoretical hypothesis, J. Electroanal. Chem. 350 (1989) 57-62.

[11] C. Siclen, S.E. Jones, Plasmons enhance fusion, J. Phys. G. Nucl. Phys. 12 (1986) 213-221.

[12] M. Baldo, R. Pucci, Nuclear matter properties from a separable representation of the Paris potential, Phys. Rev. 41 (1990) 1748-1761

[13] D.R.O. Morrison, Review of cold fusion, in: 8th World Hydrogen Energy Conference 1990 Honolulu, HI: Hawaii Natural Energy Institute, Honolulu, 1990. 